\title{
Azithromycin enhances the favorable results of paclitaxel and cisplatin in patients with advanced non-small cell lung cancer
}

\author{
D.J. Chu, D.E. Yao, Y.F. Zhuang, Y. Hong, X.C. Zhu, Z.R. Fang, \\ J. Yu and Z.Y. Yu \\ Department of Respiratory Medicine, \\ The Eighth People's Hospital of Shanghai, Shanghai, China \\ Corresponding author: D.J. Chu \\ E-mail:djchu@sina.com
}

Genet. Mol. Res. 13 (2): 2796-2805 (2014)

Received May 14, 2013

Accepted December 6, 2013

Published April 14, 2014

DOI http://dx.doi.org/10.4238/2014.April.14.8

\begin{abstract}
Although new chemotherapeutic drugs have been applied constantly, their efficacy for non-small cell lung cancer (NSCLC) is still not satisfactory. In recent years, epidemiological investigations have shown that lung cancer may be induced by chronic Chlamydia pneumoniae ( $\mathrm{Cpn}$ ) infection, since stable high titers of Cpn antibodies, especially IgA, are a hallmark of chronic infections. Azithromycin is commonly used for the treatment of Cpn infections; however, there are only few reports regarding the application of azithromycin (A) combined with paclitaxel and cisplatin (TP) for advanced NSCLC. Considering that patients with NSCLC have a higher rate of Cpn infection, we proposed to employ azithromycin for Cpn infection in chemotherapy for advanced NSCLC. The aim of this study was to explore the effects of azithromycin on chemotherapy for NSCLC. A total of 86 patients with stage III-IV NSCLC were randomly divided into TP and ATP groups; the characteristics of patients in the two groups showed no significant differences. The TP group was treated with paclitaxel and cisplatin, and the ATP group was treated with
\end{abstract}


azithromycin combined with TP for at least 4 weeks, followed by evaluation and comparison of efficacy, side effects and patients' quality of life before and after chemotherapy between the two groups. Testing for Cpn infection revealed a significant difference in the case number before and after therapy in the ATP group $(\mathrm{P}<0.01)$ compared with the TP group $(\mathrm{P}>0.05)$, and a statistical difference was observed $(\mathrm{P}<0.01)$ between the ATP and TP groups after treatment. The changes in quality of life of patients after two different chemotherapy regimens were statistically significant $(\mathrm{P}<0.05)$, but there was a significant difference in only cognitive function after treatment. The changes in symptom scores of patients after the two different chemotherapy regimens were statistically significant $(\mathrm{P}<0.05)$, but there was a significant difference in only shortness of breath and cough after treatment. Kaplan-Meier estimate was utilized to describe the survival function of patients in the two groups. The median survival time was 12.0 months for the TP group and 13.0 months for the ATP group. One-year survival rates of the TP and ATP groups were 45.0 and $75.0 \%$, respectively, which were significantly different $(\mathrm{P}<0.05)$. Our study of azithromycin+paclitaxe $1+$ cisplatin on stage III-IV NSCLC patients achieved favorable results in terms of side effects and overall survival.

Key words: Non-small cell lung cancer (NSCLC); Cisplatin; Paclitaxel; Chlamydia pneumoniae; Azithromycin

\section{INTRODUCTION}

Lung cancer, from which more than one million people in the world die every year, has been recognized as the most common cause of cancer deaths (Guilbert, 2003; Yang et al., 2010; Wang et al., 2013). Non-small cell lung cancer (NSCLC) accounts for $80 \%$ of lung cancer cases, and approximately two-thirds of patients with NSCLC are diagnosed at stage III or IV, when the cancer cannot be resected. Recently, progress has been made in early and late lung cancer, but most patients are still unable to be cured. At present, chemotherapy is the major measure against cancer. Although new chemotherapeutic drugs are applied constantly, their efficacy against NSCLC is still not satisfactory.

Some researchers believe that the initiation of lung cancer closely correlates with Chlamydia pneumoniae (Cpn) infection (Littman et al., 2005). Cpn infection is considered as the one of the dangerous factors for lung cancer (Zhan et al., 2011). To assess this relationship more precisely, a meta-analysis was performed. Twelve studies, involving 2595 lung cancer cases and 2585 controls from four prospective studies and eight retrospective studies were included. Overall, people exposed to Cpn infection had an odds ratio $(\mathrm{OR})=1.48$ and $95 \%$ confidence interval $(\mathrm{CI})=1.32-1.67$ for lung cancer risk, relative to those not exposed. Cpn infection was clearly identified as a risk factor for lung cancer in both prospective studies $(\mathrm{OR}=1.16 ; 95 \% \mathrm{CI}$ $=1.00-1.36)$ and retrospective studies $(\mathrm{OR}=2.17 ; 95 \% \mathrm{CI}=1.79-2.63)$ and in both the $\mathrm{IgA} \geq$ 16 cutoff group $(\mathrm{OR}=1.22 ; 95 \% \mathrm{CI}=1.06-1.41)$ and $\mathrm{IgA} \geq 64$ cutoff group $(\mathrm{OR}=2.35 ; 95 \% \mathrm{CI}$ $=1.88-2.93)$. In conclusion, $\mathrm{Cpn}$ infection is associated with an increased risk for lung cancer, 
and higher titers may be a better predictor of lung cancer risk. Earlier, we established a lung cancer model by repeatedly infecting Wistar rats with C. pneumoniae (Chu et al., 2012).

In recent years, great progress has been made in the chemotherapy for advanced NSCLC along with the application of third-generation drugs, represented by navelbine and paclitaxel, etc., and their combination with cisplatin. Azithromycin is commonly used for the treatment of Cpn infections, and therefore, we proposed to employ azithromycin for Cpn infection in advanced NSCLC, aiming to improve the existing treatments. However, there are only few reports regarding the application of azithromycin (A) combined with paclitaxel and cisplatin (TP) for advanced NSCLC. Therefore, our research explored the clinical effects of TP and ATP for advanced NSCLC and the results are reported herein.

\section{MATERIAL AND METHODS}

\section{Subjects}

A total of 86 patients in the Department of Respiratory Medicine were diagnosed with stage III-IV advanced NSCLC according to pathological histology between February 2008 and December 2012. These patients included 42 males and 44 females, 38 to 78 years, with a median age of 57 years. There were 35 cases at stage III and 51 cases at stage IV. This study involved 45 new patients and 41 retreatment patients, consisting of 49 cases of adenocarcinoma, 35 cases of squamous carcinoma and two cases of large cell carcinoma. These selected patients were randomly divided into the TP group and the azithromycin combined with paclitaxel and cisplatin (ATP) group. No significant differences $(\mathrm{P}>0.05)$ in gender, age, Eastern Cooperative Oncology Group (ECOG) score, pathological type, clinical stage, and metastasis of the patients were observed between the two groups (Table 1).

\begin{tabular}{|c|c|c|c|c|c|c|c|c|c|c|c|c|}
\hline \multirow[t]{2}{*}{ Regimen } & \multirow[t]{2}{*}{ Cases } & \multicolumn{2}{|c|}{ Gender } & \multirow[t]{2}{*}{ Median age } & \multicolumn{2}{|c|}{ Stages } & \multicolumn{2}{|c|}{ Former treatment } & \multicolumn{3}{|c|}{ Pathology } & \multirow[t]{2}{*}{ Cpn-IgA (+) (\%) } \\
\hline & & $\mathrm{M}$ & $\bar{F}$ & & III & $\overline{\text { IV }}$ & Primary & Secondary & $\overline{\mathrm{Sq}}$ & $\mathrm{Ad}$ & $\bar{L}$ & \\
\hline$\overline{\mathrm{TP}}$ & 43 & 21 & 22 & 57 & 17 & 26 & 23 & 20 & 17 & 25 & 1 & 51.2 \\
\hline ATP & 43 & 21 & 22 & 57 & 18 & 25 & 22 & 21 & 18 & 24 & 1 & 48.8 \\
\hline
\end{tabular}

$\mathrm{Sq}=$ squamous carcinoma; $\mathrm{Ad}=$ adenocarcinoma; $\mathrm{L}=$ large cell carcinoma; $\mathrm{TP}=$ paclitaxel and cisplatin; ATP $=$ azithromycin combined with paclitaxel and cisplatin.

\section{Inclusion criteria}

The patients had stage III or IV NSCLC and a Karnofsky score $>70$, and had received at least one cycle of chemotherapy including platinum drugs, followed by disease progression or recurrence. The interval between last chemotherapy and our treatment was more than 28 days, and there had been complete recovery from the side effects of previous chemotherapy. Patients were 38 to 78 years old with normal hematological and hepatorenal functions. Expected survival was more than three months, and efficacy evaluation of the treatments was available. There was no brain metastasis. All patients signed an informed consent before treatment and were approved by the hospital Ethics Committee. 


\section{Exclusion criteria}

Patients were excluded if they had only undetectable lesions, such as metastasis of abdominal and thoracic effusions or bone metastasis. Also excluded where patients with brain metastasis, those with serious infections or uncured second primary malignant tumor, retreatment patients who took azithromycin or paclitaxel in previous chemotherapy, and those with mental disorder.

\section{Preparation of serum sampling and Cpn-specific antibody test}

The venous blood samples from lung cancer patients were collected in the morning on an empty stomach. The blood samples from the patients who were undergoing chemotherapy were collected before and 1 week after chemotherapy. After centrifugation for $5 \mathrm{~min}$, the serum was isolated and preserved at $-20^{\circ} \mathrm{C}$ for further tests. Cpn-specific antibodies (Cpn-IgA, -IgG and - IgM) were measured using the MIF technique. Positive and negative controls were included in every test and the results were verified by two professionals under a fluorescent microscope.

\section{Treatment strategy}

All 86 NSCLC patients between February 2008 and December 2012 were randomly divided into two groups. The patients in the TP group were injected with $175 \mathrm{mg} /$ $\mathrm{m}^{2}$ paclitaxel for at least $3 \mathrm{~h}$ each time for six cycles with an interval of 3 weeks between each cycle (Schallier et al., 2007). Venous infusion with $60 \mathrm{mg} / \mathrm{m}^{2}$ cisplatin was also performed for 4 weeks as one cycle. Evaluation of efficacy was conducted after four cycles of treatment, followed by verification of therapeutic efficiency 6 weeks after treatment. The patients in the ATP group took $500 \mathrm{mg}$ azithromycin orally per day for 5 consecutive days and three cycles with an interval of 23 days (one cycle lasted 28 days) (Brandén et al., 2004). In addition, paclitaxel and cisplatin were also given to the patients in the ATP group like those in the TP group. Electrocardiogram, chest radiograph, chest CT, or bronchofiberscope biopsy and liver, kidney function were reexamined after chemotherapy. A hemogram was done twice a week.

\section{Efficacy evaluation criterion and observation indicators}

Major indicators included response rate and time to progression, and minor indicators consisted of quality of life (QOL) and the safety and toxicity of drug. Short-term efficacy was evaluated according to the objective standard of WHO for antineoplastic drugs, including complete response (CR), partial response (PR), stable disease, and progressive disease. Long-term efficacy indicators were overall survival rate and median survival time.

\section{Follow-up visit}

All patients were followed up until December 20, 2012 and the median follow-up time was 25 months. 


\section{Statistical analyses}

STATA 10.0 was utilized to perform statistical analysis. A paired $t$-test was conducted to compare QOL and the symptom score before and after treatment. An R test was performed to assess the relationship of efficacy with QOL and symptom-improved efficiency. Singlefactor analysis of survival rate was performed using the Kaplan-Meier method. Comparison of toxic and side effects between the two groups was conducted using the chi-square test. $\mathrm{P} \leq$ 0.05 was considered to be statistically significant.

\section{RESULTS}

\section{Comparison of the number of $\mathrm{Cpn}$ infective cases before and after treatment between the two groups}

There was no statistical difference $(\mathrm{P}>0.05)$ in the number of $\mathrm{Cpn}-\operatorname{IgA}(+), \mathrm{Cpn}-$ $\operatorname{IgG}(+)$ and $\mathrm{Cpn}-\operatorname{IgM}(+)$ infective cases in the TP group before and after the therapy, while the number of $\mathrm{Cpn}-\operatorname{IgA}(+), \mathrm{Cpn}-\operatorname{IgG}(+)$ and $\mathrm{Cpn}-\operatorname{IgM}(+)$ infective cases in the ATP group was significantly different $(\mathrm{P}<0.01)$ before and after the therapy. The difference in number of Cpn-IgA(+), Cpn-IgG(+) and Cpn-IgM(+) infective cases between the TP and ATP groups was significant $(\mathrm{P}<0.05)$ after therapy. The details are shown in Table 2.

\begin{tabular}{|c|c|c|c|c|c|c|c|c|c|c|}
\hline \multirow[t]{2}{*}{ Regimen } & \multirow[t]{2}{*}{ Cases } & \multicolumn{2}{|c|}{ Cpn- $\operatorname{IgA}(+)$} & \multirow[t]{2}{*}{$\mathrm{P}^{*}$} & \multicolumn{2}{|c|}{ Cpn-IgG(+) } & \multirow[t]{2}{*}{$\mathrm{P}^{*}$} & \multicolumn{2}{|c|}{ Cpn-IgM(+) } & \multirow[t]{2}{*}{$\mathrm{P}^{*}$} \\
\hline & & Pre- & Post- & & Pre- & Post- & & Pre- & Post- & \\
\hline TP & 43 & 22 & 20 & 0.666 & 24 & 19 & 0.281 & 13 & 10 & 0.465 \\
\hline TP & 43 & 21 & 10 & 0.013 & 22 & 10 & 0.007 & 11 & 3 & 0.019 \\
\hline $\mathrm{P}^{* *}$ & & $>0.05$ & 0.024 & & $>0.05$ & 0.040 & & $>0.05$ & 0.035 & \\
\hline
\end{tabular}

Pre- = pre-treatment; Post- $=$ post-treatment; $* \mathrm{P}$ for statistical results of comparison between pre- and posttreatments at the same set of objects. **P for statistical results of comparison between TP and ATP groups after treatment. For abbreviations, see Table 1.

\section{Comparison of quality of life and symptom score of patients in the two groups}

The changes in QOL of patients after the two different chemotherapy regimens were significantly different $(\mathrm{P}<0.05)$ from those before the treatment in the same group, but only in the cognitive function there was a significant difference $(\mathrm{P}<0.05)$ after treatment between the TP and ATP groups. The details are shown in Table 3.

Table 3. Changes of quality of life in patients after the two different chemotherapy regimens.

\begin{tabular}{|c|c|c|c|c|c|c|c|}
\hline \multirow[t]{2}{*}{ Project } & \multicolumn{2}{|c|}{$\mathrm{TP}$ group $(\mathrm{N}=43)$} & \multirow[t]{2}{*}{$\mathrm{P} *$} & \multicolumn{2}{|c|}{ ATP group $(\mathrm{N}=43)$} & \multirow[t]{2}{*}{$\mathrm{P} *$} & \multirow[t]{2}{*}{$\mathrm{P} * *$} \\
\hline & Pre-treatment & Post-treatment & & Pre-treatment & Post-treatment & & \\
\hline Overall situation & $40.25 \pm 11.35$ & $45.78 \pm 8.19$ & 0.011 & $39.91 \pm 10.24$ & $44.36 \pm 9.25$ & 0.0292 & $>0.05$ \\
\hline Physical function & $46.14 \pm 12.62$ & $52.65 \pm 11.38$ & 0.014 & $45.87 \pm 12.42$ & $52.21 \pm 12.33$ & 0.0198 & $>0.05$ \\
\hline Emotional function & $61.62 \pm 13.19$ & $67.26 \pm 10.33$ & 0.030 & $62.15 \pm 13.61$ & $69.25 \pm 14.63$ & 0.0222 & $>0.05$ \\
\hline Cognitive function & $63.26 \pm 7.02$ & $67.12 \pm 8.91$ & 0.028 & $63.84 \pm 6.89$ & $72.42 \pm 10.48$ & 0.0001 & 0.033 \\
\hline Social function & $43.21 \pm 12.23$ & $49.62 \pm 13.36$ & 0.037 & $44.83 \pm 12.92$ & $50.61 \pm 13.13$ & 0.0427 & $>0.05$ \\
\hline
\end{tabular}

*P for statistical results of comparison between pre- and post-treatments on the same objects; **P for statistical results of comparison between TP and ATP groups after treatment. For abbreviations, see Table 1. 


\section{Comparison of symptom score of patients in the two groups}

The changes in symptom scores of patients were statistically significant $(P<0.05)$ compared to before treatment in the same group, but only the changes in cough and shortness of breath after treatment differed significantly $(\mathrm{P}<0.05)$ between the TP and ATP groups, with the changes in other symptom scores showing no difference $(\mathrm{P}>0.05)$. The details are shown in Table 4.

\begin{tabular}{|c|c|c|c|c|c|c|c|}
\hline \multirow[t]{2}{*}{ Project } & \multicolumn{2}{|c|}{ TP group $(\mathrm{N}=43)$} & \multirow[t]{2}{*}{$\mathrm{P}^{*}$} & \multicolumn{2}{|c|}{ ATP group $(\mathrm{N}=43)$} & \multirow[t]{2}{*}{$\mathrm{P}^{*}$} & \multirow[t]{2}{*}{$\mathrm{P} * *$} \\
\hline & Pre-treatment & Post-treatment & & Pre-treatment & Post-treatment & & \\
\hline Fatigue & $55.63 \pm 10.32$ & $50.98 \pm 10.09$ & 0.038 & $56.19 \pm 10.86$ & $50.84 \pm 8.23$ & 0.012 & $>0.05$ \\
\hline Anorexia & $42.58 \pm 8.77$ & $38.63 \pm 7.91$ & 0.031 & $42.88 \pm 9.24$ & $38.49 \pm 7.72$ & 0.019 & $>0.05$ \\
\hline Shortness of breath & $58.52 \pm 13.15$ & $51.72 \pm 8.61$ & 0.049 & $58.09 \pm 8.46$ & $47.79 \pm 7.89$ & 0.001 & 0.030 \\
\hline Cough & $49.56 \pm 8.76$ & $45.35 \pm 7.66$ & 0.020 & $49.37 \pm 11.28$ & $39.93 \pm 7.74$ & 0.001 & 0.002 \\
\hline Pain & $33.61 \pm 6.18$ & $28.58 \pm 5.89$ & 0.001 & $33.74 \pm 6.66$ & $28.12 \pm 5.56$ & 0.001 & $>0.05$ \\
\hline
\end{tabular}

*P for statistical results of comparison between pre- and post-treatments on the same regimen. **P for statistical results of comparison between TP and ATP groups after treatment. For abbreviations, see Table 1.

\section{Evaluation of efficacy}

The response rates were $32.5 \%(2.3 \% \mathrm{CR}$ and $30.2 \% \mathrm{PR})$ in the TP group and $67.5 \%$ $(4.7 \% \mathrm{CR}$ and $62.8 \% \mathrm{PR})$ in the ATP group, which were significantly different $(\mathrm{P}>0.05)$ between the TP and ATP groups. The median survival time was 12.0 months in the TP group and 13.0 months in the ATP group. One-year survival rates of the TP and ATP groups were 45.0 and $75.0 \%$ respectively, which were significantly different $(\mathrm{P}<0.05)$. The details are shown in Table 5.

Table 5. Comparison of efficacy of the two different chemotherapy regimens.

\begin{tabular}{|c|c|c|c|c|c|c|c|c|}
\hline \multirow[t]{2}{*}{ Regimen } & \multirow[t]{2}{*}{ Cases } & \multicolumn{4}{|c|}{ Evaluation of efficacy } & \multirow[t]{2}{*}{ Months $^{\mathrm{a}}$} & \multirow[t]{2}{*}{1 year $(\%)^{b}$} & \multirow[t]{2}{*}{2 years $(\%)^{c}$} \\
\hline & & CR (\%) & PR (\%) & $\mathrm{SD}(\%)$ & PD (\%) & & & \\
\hline $\mathrm{TP}$ & 43 & $1(2.3)$ & $13(30.2)$ & $17(39.5)$ & $12(20.9)$ & 12.0 & 45.0 & 18.0 \\
\hline ATP & 43 & $2(4.7)$ & $27(62.8)$ & $9(20.9)$ & 5 (11.6) & 13.0 & 75.0 & 20.0 \\
\hline $\mathrm{P}^{*}$ & 0.001 & & & 0.001 & & $>0.05$ & 0.040 & $>0.05$ \\
\hline
\end{tabular}

$\mathrm{CR}=$ complete response; $\mathrm{PR}=$ partial response; $\mathrm{SD}=$ stable disease $; \mathrm{PD}=$ progressive disease; ${ }^{\text {arepresent }}$ median

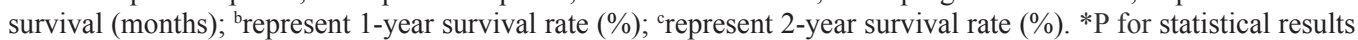
of comparison between TP and ATP groups after treatment.

\section{Survival curve}

Two-year survival rates of the TP and ATP groups were 18.0 and $20.0 \%$, respectively $(\mathrm{P}>0.05)$. Kaplan-Meier estimate was utilized to describe the survival function of patients in the two groups: $\chi^{2}(1)=4.23, \mathrm{P}>\chi^{2}=0.0398$ (Figure 1). 


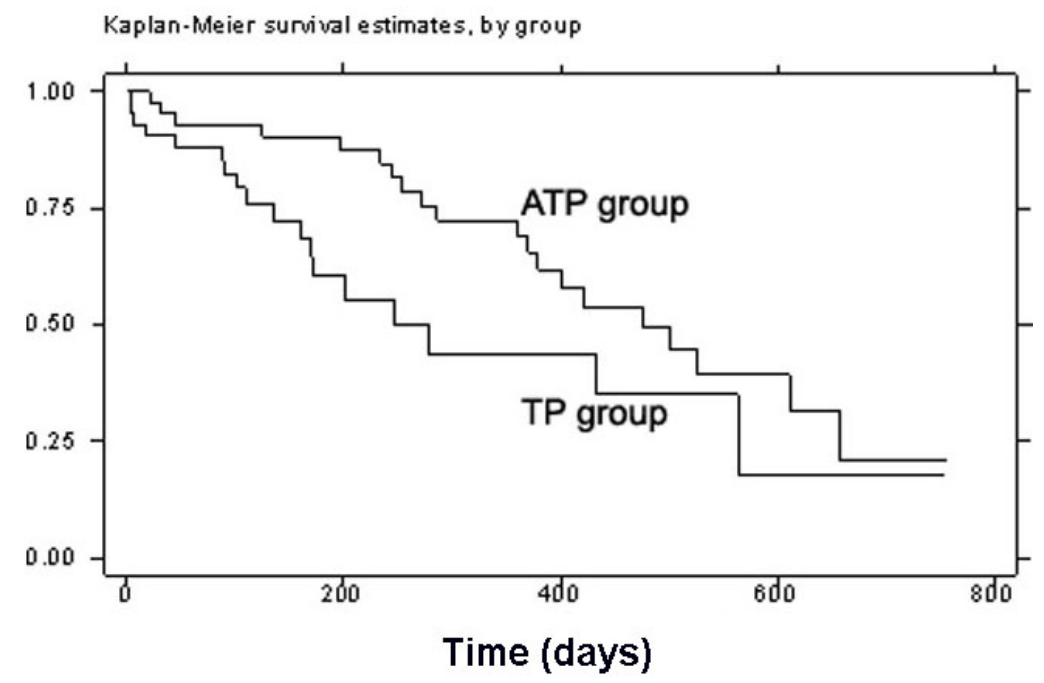

Figure 1. Kaplan-Meier survival curve of patients with advanced NSCLC. Kaplan-Meier estimate was utilized to describe the survival function of patients in ATP and TP groups: $\chi^{2}(1)=4.23, \operatorname{Pr}>\chi^{2}=0.0398$. One-year survival rate of TP and ATP groups were 45.0 and $75.0 \%$, respectively, which were significantly different $(\mathrm{P}<0.05)$. Twoyear survival rates of TP and ATP groups were 18.0 and $20.0 \%$, respectively $(\mathrm{P}>0.05)$.

\section{Comparison of the incidence of side effects}

The main side effects were bone marrow suppression, where the incidence of leukopenia, neutropenia and thrombocytopenia was $62.8,72.1$ and $16.3 \%$ in the TP group, and 65.1, 74.4 and $18.6 \%$ in the ATP group, respectively. The incidence of nausea/vomiting and fatigue between TP and ATP groups showed a significant difference $(\mathrm{P}<0.05)$. The other side effects showed no statistical significance $(\mathrm{P}>0.05)$. All side effects are shown in Table 6 .

\begin{tabular}{|c|c|c|c|c|c|c|c|}
\hline \multirow[t]{2}{*}{ Side effect } & \multicolumn{2}{|c|}{$\mathrm{TP}(\mathrm{N}=43)$} & \multirow[t]{2}{*}{ No. (\%) } & \multicolumn{2}{|c|}{$\operatorname{ATP}(\mathrm{N}=43)$} & \multirow[t]{2}{*}{ No. $(\%)$} & \multirow[t]{2}{*}{$\mathrm{P}^{*}$} \\
\hline & $\begin{array}{c}\alpha-\alpha \\
(\mathrm{N}=14)\end{array}$ & $\begin{array}{c}\beta-\chi \\
(\mathrm{N}=29) \\
\end{array}$ & & $\begin{array}{c}\alpha-\alpha \\
(\mathrm{N}=29) \\
\end{array}$ & $\begin{array}{c}\beta-\chi \\
(\mathrm{N}=14)\end{array}$ & & \\
\hline \multicolumn{8}{|l|}{ Myelosupression } \\
\hline Leucopenia & 12 & 15 & $27(62.8)$ & 17 & 11 & $28(65.1)$ & 0.822 \\
\hline Neutropenia & 13 & 18 & $31(72.1)$ & 19 & 13 & $32(74.4)$ & 0.808 \\
\hline Thrombocytopenia & 4 & 3 & $7(16.3)$ & 5 & 3 & 8 (18.6) & 0.776 \\
\hline \multicolumn{8}{|l|}{ Digestive tract } \\
\hline Nausea/vomiting & 5 & 5 & $10(23.3)$ & 1 & 2 & $3(7.0)$ & 0.035 \\
\hline Stomatitis & 1 & 1 & $2(4.7)$ & 1 & 2 & $3(7.0)$ & 0.645 \\
\hline Wind/constipation & 0 & 1 & $1(2.3)$ & 1 & 1 & $2(4.7)$ & 0.557 \\
\hline \multicolumn{8}{|l|}{ Others } \\
\hline Fatigue & 5 & 6 & $11(25.6)$ & 1 & 2 & $3(7.0)$ & 0.019 \\
\hline Phlebitis & 1 & 2 & $3(7.0)$ & 1 & 3 & $4(9.3)$ & 0.693 \\
\hline Peripheral neuropathy & 1 & 1 & $2(4.7)$ & 1 & 2 & $3(7.0)$ & 0.645 \\
\hline Alopecia & 1 & 1 & $2(4.7)$ & 1 & 3 & $4(9.3)$ & 0.397 \\
\hline
\end{tabular}

*P for statistical results of comparison between TP and ATP groups. 


\section{DISCUSSION}

Platinum-based combination with third-generation drugs has become the standard therapy for advanced NSCLC, but there are only few published papers with respect to randomized controlled studies of platinum triplet regimen. Because the patients with NSCLC had a Cpn infection rate of 53.5\% (46/86), the ones with advanced NSCLC in our study were randomly treated with TP or ATP. The numbers of Cpn-IgA(+), Cpn-IgG(+) and Cpn-IgM(+) infective cases in the ATP group significantly changed $(\mathrm{P}<0.01)$ after the therapy. The difference in numbers of Cpn-IgA(+), Cpn-IgG(+) and Cpn-IgM(+) infective cases between the TP and ATP groups was significant $(\mathrm{P}<0.05)$ after therapy. Meanwhile, the response rate was $32.5 \%(2.3 \% \mathrm{CR}$ and $30.2 \% \mathrm{PR})$ in the TP group, which was significantly different $(\mathrm{P}<0.05)$ from $67.5 \%(4.7 \% \mathrm{CR}$ and $62.8 \% \mathrm{PR})$ in the ATP group. The median survival time was 12.0 months in the TP group and 13.0 months in the ATP group. One-year survival rates of the TP and ATP groups were 45.0 and $75.0 \%$, respectively, which were significantly different $(\mathrm{P}<$ $0.05)$. The main side effects was bone marrow suppression, where the incidence of leukopenia, neutropenia and thrombocytopenia was $62.8,72.116 .3 \%$ in the TP group and 65.1, 74.4 $18.6 \%$ in the ATP group, respectively. The incidence of nausea/vomiting and fatigue between the TP and ATP groups was significantly different $(\mathrm{P}<0.05)$. The patients of the ATP group displayed improved quality of life compared with that of the TP group, but no statistical difference was observed between the two groups. Kaplan-Meier estimate was utilized to describe the survival function of patients in the two groups: $\chi^{2}(1)=4.23, \operatorname{Pr}>\chi^{2}=0.0398$. All results indicated that the treatment efficacy in the TP group was superior to that in the ATP group. In a word, the toxic effects of the two chemotherapies were mild and were eliminated by symptomatic treatment; serious hematologic toxicity was not observed. On the basis of these results derived from randomized trials, it appears that advanced NSCLC patients with goodperformance status should be treated with regimens containing azithromycin in conjunction with paclitaxel and cisplatin (ATP).

At present, cisplatin has always been applied in the treatment for advanced NSCLC, which induces cancer cell apoptosis by damaging DNA and activating signaling pathway of cell apoptosis (Siddik, 2003; Jiang et al., 2011). Paclitaxel, a new type of microtubule stabilizer, is able to inhibit cancer cell growth due to its unique mechanism: promoting tubulin polymerization and making depolymerization difficult. Paclitaxel specifically catalyzes tubulin synthesis and binding to the microtubules during cell mitosis, which makes the spindle lose normal function and prevents cancer cell proliferation. Other research has discovered that paclitaxel induces macrophages to release a series of tumor-killing cells or cytokines that inhibit the metastasis of tumor cells, as well as suppressing tumor angiogenesis, which is key for tumor growth (Jiang et al., 2010). Therefore, TP chemotherapy significantly improves the symptoms in patients with NSCLC, but it is unable to prolong the overall response rate and overall survival of patients.

Azithromycin is a new generation of macrolide antibiotics, which is also one of the primary drugs for the treatment of Cpn infection. Azithromycin has besides an antibacterial effect an anti-inflammatory action, whose mechanism is now considered to involve the following aspects (Wuyts et al., 2010): inhibition of mucin secretion from airway epithelia and decrease in secretion of airway mucus by blocking $\mathrm{Cl}^{-}$channels and suppressing the excretion of water; inhibition of IL-8 secretion from airway epithelia and of LT-B4 release from 
neutrophils and decrease in neutrophil recruitment in the airway. It also exerts potential immunomodulatory effects. Daenas et al. (2006) believed that the application of azithromycin was beneficial in treating Cpn-infected patients, since azithromycin could directly relax the contracting airway smooth muscle, which could improve the breathing situation of patients. IgA has a half-life of 5-6 days and decreases after acute infection, thus continuous IgA rising is considered to be the best indicator of chronic infection. IgM antibodies appear with a high titer 1 weeks after the first infection by Cpn, while $\operatorname{IgG}$ appears 6 to 8 weeks later. However, IgM antibodies do not show up or only have low titer in the second infection, but IgG level rapidly increases in 1 to 2 weeks (titer is higher than 1:512), which can be sustained for years. In addition, IgM extensively exists in humans and its positive rate increases with age. Cpn infection has chronic, persistent tendency, which may result from the fact that Cpn inhibits its apoptosis by blocking the release of cytochrome $\mathrm{c}$ from mitochondria of host cells and caspase-3 activity. Kocazeybek et al. (2003) reported that the titers of Cpn-IgA and Cpn-IgG in lung cancer patients could be higher than 1:40 and 1:512, respectively; they found a Cpn infection rate of 50.4\% in general cases, which indicated that chronic Cpn infection enhanced the risk of lung cancer. Chu et al. (2012) confirmed this opinion through the establishment of a lung cancer model by repeatedly infecting Wistar rats with Cpn. Although there is dispute regarding the relationship of $\mathrm{Cpn}$ infection and lung cancer, $\mathrm{Cpn}$ infection rate in patients with lung cancer is obviously higher than that of healthy people, and these patients benefit from the application of azithromycin.

But above all, our study of azithromycin+paclitaxel+cisplatin in stage III-IV NSCLC patients achieved favorable results in terms of toxicity and overall survival. ATP chemotherapy is recommended for lung cancer patients with chronic Cpn infection, but the long-term effects of azithromycin on lung cancer patients, including long-term improvement of symptoms and survival, require further investigations.

\section{REFERENCES}

Brandén E, Koyi H, Gnarpe J, Gnarpe H, et al. (2004). Intermittent azithromycin treatment for respiratory symptoms in patients with chronic Chlamydia pneumoniae infection. Scand. J. Infect. Dis. 36: 811-816.

Chu DJ, Guo SG, Pan CF, Wang J, et al. (2012). An experimental model for induction of lung cancer in rats by Chlamydia pneumoniae. Asian Pac. J. Cancer Prev. 13: 2819-2822.

Daenas C, Hatziefthimiou AA, Gourgoulianis KI and Molyvdas PA (2006). Azithromycin has a direct relaxant effect on precontracted airway smooth muscle. Eur. J. Pharmacol. 553: 280-287.

Guilbert JJ (2003). The world health report 2. Educ. Health 16: 230.

Jiang B, Zhu ZZ, Liu F, Yang LJ, et al. (2011). STAT3 gene polymorphisms and susceptibility to non-small cell lung cancer. Genet. Mol. Res. 10: 1856-1865.

Jiang H, Tao W, Zhang M, Pan S, et al. (2010). Low-dose metronomic paclitaxel chemotherapy suppresses breast tumors and metastases in mice. Cancer Invest. 28: 74-84.

Kocazeybek B (2003). Chronic Chlamydophila pneumoniae infection in lung cancer, a risk factor: a case-control study. J. Med. Microbiol. 52: 721-726.

Littman AJ, Jackson LA and Vaughan TL (2005). Chlamydia pneumoniae and lung cancer: epidemiologic evidence. Cancer Epidemiol. Biomarkers Prev. 14: 773-778.

Schallier D, Neyns B, Fontaine C, Steene JV, et al. (2007). A novel triplet regimen with paclitaxel, carboplatin and gemcitabine (PACCAGE) as induction chemotherapy for locally advanced unresectable non small cell lung cancer (NSCLC). Lung Cancer 56: 247-254.

Siddik ZH (2003). Cisplatin: mode of cytotoxic action and molecular basis of resistance. Oncogene 22: 7265-7279.

Wang H, Zhao Y, Ma J, Zhang G, et al. (2013). The genetic variant rs401681C/T is associated with the risk of non-small cell lung cancer in a Chinese mainland population. Genet. Mol. Res. 12: 67-73. 
Wuyts WA, Willems S, Vos R, Vanaudenaerde BM, et al. (2010). Azithromycin reduces pulmonary fibrosis in a bleomycin mouse model. Exp. Lung Res. 36: 602-614.

Yang L, Shi GL, Song CX and Xu SF (2010). Relationship between genetic polymorphism of MCP-1 and non-small-cell lung cancer in the Han nationality of North China. Genet. Mol. Res. 9: 765-771.

Zhan P, Suo LJ, Qian Q, Shen XK, et al. (2011). Chlamydia pneumoniae infection and lung cancer risk: a meta-analysis. Eur. J. Cancer 47: 742-747. 